\title{
Exponential Time/Space Speedups for Resolution and the PSPACE-completeness of Black-White Pebbling
}

\author{
Philipp Hertel* \\ Department of Computer Science \\ University of Toronto \\ Toronto, ON CANADA \\ philippecs.toronto.edu
}

\author{
Toniann Pitassi* \\ Department of Computer Science \\ University of Toronto \\ Toronto, ON CANADA \\ toni@cs.toronto.edu
}

\begin{abstract}
The complexity of the Black-White Pebbling Game has remained open for 30 years. It was devised to capture the power of non-deterministic space bounded computation. Since then it has been applied to problems in diverse areas of computer science including VLSI design and more recently propositional proof complexity. In this paper we show that the Black-White Pebbling Game is PSPACE-complete. We then use similar ideas in a more complicated reduction to prove the PSPACEcompleteness of Resolution space. The reduction also yields a surprising exponential time/space speedup for Resolution in which an increase of 3 units of space results in an exponential decrease in proof-size.
\end{abstract}

\section{Introduction}

The Black-White Pebbling Game was introduced by Cook and Sethi in 1976 [3] in the context of determining lower bounds for space bounded Turing Machines. The problem recevied considerable attention throughout the next decade due to its numerous applications including VLSI design, compilers, and algebraic complexity. In 1983 determining its complexity was rated as "An Open Problem of the Month" in David Johnson's NPCompleteness Column [9]. An excellent survey of pebbling results from this period can be found in Pippenger [14]. Recently, there has been a resurgence of interest in pebbling games due to their links with propositional proof complexity $[2,5,12]$. In this paper we prove that the Black-White Pebbling Game is PSPACE-complete.

The Black-White Pebbling Game was preceded by

*Funded in part by NSERC the Black Pebbling Game, which has also been widely studied [14]. Let $\mathcal{G}=(V, E)$ be a DAG with one distinguished output node, $s$. In the Black Pebbling Game, a player tries to place a pebble on $s$ while minimizing the number of pebbles placed simultaneously on $\mathcal{G}$. The game is split up into distinct steps, each of which takes the player from one pebbling configuration to the next. Initially, the graph contains no pebbles and each subsequent configuration follows from the previous by one of the following rules: 1) At any point a black pebble can be placed on any source node v. 2) At any point a black pebble can be removed from any node $v$. 3) For any node $v$, if all of $v$ 's predecessors have pebbles on them, then a black pebble can be placed on $v$, or a black pebble can be slid from a predecessor $u$ to $v$.

The Black Pebbling Game models deterministic space-bounded computation. Each node models a result and the placement of a black pebble on a node represents the deterministic computation of the result from previously computed results. A sequence of moves made by the player is called a pebbling strategy. If a strategy manages to pebble $s$ using no more than $k$ pebbles, then that strategy is called a $k$-pebbling strategy.

The Black-White Pebbling Game is a more powerful extension of the Black Pebbling Game in which white pebbles, which behave in a dual manner to the original black pebbles, can also be used. As before, the player attempts to place a black pebble on $s$ while minimizing the number of pebbles placed simultaneously on $\mathcal{G}$ at any time. The Black-White Pebbling Game extends the Black Pebbling Game with the addition of the following rules: 4) At any point a white pebble can be placed on any node $v$. 5) At any point a white pebble can be removed from any source node $v$. 6) For any node $v$ with a white pebble on it, the pebble can be slid to an empty predecessor $u$ if all of $v$ 's other predecessors are pebbled, 
or the white pebble can be removed if all of v's predecessors are pebbled. 7) The game ends when $s$ contains a black pebble and every other node is empty.

As before, the placement of each black pebble is meant to model the derivation of a deterministicallycomputed result, while the placement of each white pebble is meant to model a non-deterministic guess, whose verification requires all of its antecedents to be derived.

In 1978, Lingas showed that a generalization of the Black Pebbling Game, played on monotone circuits instead of DAGs, is PSPACE-complete [11]. This was a surprising result since the PSPACE-complete games of the time involved two players and it was clear how the alternation between them led to each game's high complexity. In 1980, Gilbert, Lengauer, and Tarjan elaborated on the basic structure of Lingas's construction to prove that the Black Pebbling Game on DAGs is PSPACE-complete [6].

While the above results resolve the complexity of black pebbling, determining the complexity of black white pebbling has resisted numerous attempts. In contrast to black pebbling, white pebbles allow a much richer choice of strategies since they can be placed anywhere on the graph regardless of previous pebble placements, thereby breaking up the straight inductive pattern obvious in all pure black strategies. Although the black pebbling number of a graph is never more than a square of the black-white pebbling number [7], the addition of white pebbles lowers the pebbling number of many graphs [15], [10]. Unfortunately, the constructions used for the previous PSPACE-completeness results are both examples of such graphs. As a result, neither can be used to differentiate between true and false QBFs in the presence of white pebbles.

In Section 2, we finally resolve Johnson's open problem by building on the construction of [6] to prove the PSPACE-completeness of the Black-White Pebbling Game. Our reduction also provides an infinite family of graphs which require exponential time to minimally black-white pebble, but can be pebbled in linear time if we use just one pebble more than the minimum. This results in a time/space tradeoff result similar to that proved in [6] for pure black pebbling.

In Section 3, we use similar ideas in a more complicated reduction to prove the PSPACE-completeness of Resolution space as well as an exponential time/space tradeoff for Resolution. These results are motivated by recent interest in the space required by Resolution proofs and its connection to practical algorithms for solving SAT. The satisfiability problem (SAT) has become a viable and widespread approach for solving real-world problems. SAT procedures are now a standard tool for solving problems in hardware verification, circuit diagnosis, experimental design, planning and diagnosis problems. Surprisingly, the best algorithms are highly optimized variants of DPLL which is nothing more than a backtrack search for a tree-like Resolution refutation. The most successful variant, clause learning, employs a very clever type of caching scheme. It underlies all state-of-the art complete algorithms for solving SAT.

The basic idea behind clause learning is very simple: while performing the backtrack search, store intermediate clauses that are learned along the way, in order to potentially prune the remaining search space. The main issue stems from the fact that in reality there is only a finite amount of space available. Therefore, all clauses simply cannot be stored, and the difficulty is in obtaining a highly selective and efficient, yet effective caching scheme. This has inspired a great deal of research into methods and heuristics for caching schemes, resulting in state-of-the art algorithms for SAT.

Underlying most of this empirical work is an assumption that there is a smooth, nearly linear tradeoff between time and space. For example, anyspace algorithms have been developed for SAT and \#SAT where a given implementation can use as much space as is currently available [4]. They used empirical results on certain distributions of inputs to suggest that for most ranges of parameters, the tradeoff between runtime and space is nearly linear. In this paper we present theoretical results that run strongly counter to this belief.

While time/space issues for Resolution-based satisfiability algorithms have been of central importance for many years, it was only in the late nineties when the formal study of space as a complexity measure for propositional proof systems was initiated. In 1999, Esteban and Toran [5] proposed a definition of space complexity for Resolution, called clause space, that measures the number of clauses that need to be kept simultaneously in memory in order to verify the Resolution refutation.

Alekhnovich et al. [1] address the question of how to measure the memory content for more general propositional proof systems. While the most obvious choice is "bit space," [1] introduces the related notion of variable space, which counts the number of variable occurrences that must simultaneously be kept in memory. They argue that variable space and bit space are within a logarithmic factor of one another, but variable space makes the model substantially cleaner. Thus we view variable space as the right space measure to study: it applies to a variety of proof systems, and captures in a natural and clean way the space utilization of a broad range of com- 
plete algorithms for SAT.

In 2001, Ben-Sasson [2] was the first to study formal time/space tradeoffs for Resolution. ${ }^{1} \mathrm{He}$ asked if there are formulas that have optimal proofs with respect to any one of the parameters, but where optimizing one parameter must cost an increase in the other parameter. $\mathrm{He}$ proved that this is the case for tree-like Resolution. That is, he showed that there are formulas that have tree-like Resolution proofs with linear size and also have (other) tree-like Resolution proofs with constant clause space. But on the other hand, he showed that these formulas have no single tree-like Resolution proof with both linear size and constant clause space.

However, for general Resolution the problem remained open. The main result of Section 3 is an answer to this question, showing that in a very strong sense it is not possible to optimize both size and space simultaneously. We exhibit formulas that require exponential size to refute if restricted to a minimal variable space Resolution implementation, but with just three more units of space, the proof size drops to linear! In light of our earlier discussion, this result is surprising, as it runs counter to the belief is that there is a smooth,almost linear tradeoff between space and time.

We also prove a related theorem. Given a CNF formula $F$, the Resolution space problem is to determine the minimal-space Resolution proof of $F$. We prove that the Resolution space problem is PSPACE-complete, affirming that memory management for Resolution-based SAT algorithms is a complex issue.

\section{Black-White Pebbling}

\subsection{Definitions and Proof Overview}

Formally, the Black-White Pebbling Game takes as input a DAG $\mathcal{G}$ with a special target node $s$ and an integer $k$ and asks whether there is a $k$-pebbling strategy for $s$ in $\mathcal{G}$. We prove the following theorem.

Theorem 1: The Black-White Pebbling Game is PSPACE-complete.

It is not hard to see that black-white pebbling is in PSPACE. Given $(\mathcal{G}, k)$, we can easily guess a sequence of configurations that pebbles $\mathcal{G}$ with at most $k$ pebbles.

\footnotetext{
${ }^{1}$ In the algorithms literature, this tradeoff is viewed as a time/space tradeoff, whereas from a proof complexity point of view, the tradeoff would be more accurately called a size/space tradeoff. Size and time are equated because the runtime of a Resolution-based SAT algorithm is tightly connected to the size of the underlying Resolution proof.
}

Then by Savitch's theorem, this implies that black-white pebbling is in (deterministic) PSPACE.

The rest of this section is devoted to showing that the Black-White Pebbling Game is PSPACE-hard. To prove this, we will reduce from QSAT. Given a QBF $\psi$, we will create a graph $\mathcal{G}$ with the property that $\psi$ is in QSAT if and only if $\mathcal{G}$ has a $4 n+3$ black-white pebbling strategy.

Following the conventions of [13] and [6], we classify pebble placements as necessary or unnecessary. The first placement of a black pebble on the target vertex is necessary. A placement of a black or white pebble on any other node $v$ is necessary if and only if the pebble remains on $v$ until a necessary placement occurs on a successor of $v$ (this can occur concurrently if we are sliding a black pebble up from $v$ to the successor). We call a pebbling strategy which contains no unnecessary placements frugal. Clearly, removing all unnecessary placements from a $k$-pebbling strategy for a graph $G$ results in a frugal $k$-pebbling strategy for $\mathcal{G}$. We can therefore limit ourselves to considering just frugal pebblings.

Our construction is similar at a high-level to [6], where they create a graph from a QBF with the property that the formula is in QSAT if and only if the graph has a small pure black pebbling strategy. The general idea behind their reduction is to have the black pebbling correspond to the exponential-time procedure that verifies that $\psi$ is in QSAT. The graph is composed of two main parts: a linear chain of clause widgets followed by a linear chain of quantifier widgets. In all strategies which achieve the graph's minimum pebbling number, pebbles must be placed on certain special nodes in a way which corresponds to the lexicographically first truth assignment in the QSAT model for $\psi$. Since this assignment satisfies $\psi$ 's $3 \mathrm{CNF}$ the player is able to successfully pebble through the clause widgets without exceeding the minimum pebbling number. The player can then begin to make progress through the quantifier widgets up to the first universal widget, say widget $i$. In order to pebble through this widget without exceeding the pebbling number, the player must leave a pebble on a "progress node" in widget $i$ and then repebble the special nodes for the innermost $i$ variables, thereby placing pebbles in a way which corresponds to the lexicographically second truth assignment in the QSAT model. The player can then pebble up through the clause widgets again, and this time use the pebble which was previously placed on the progress node to pebble through widget $i$, only to have his/her progress arrested at the next universal widget, at which point the process must repeat. Minimally black pebbling the graph corresponding to a true QBF with $k$ universal quantifier widgets therefore requires $2^{k}$ 
time.

Unfortunately, the graphs used in all earlier constructions are easy to pebble once white pebbles are allowed, regardless of whether or not the QBF is in QSAT. Thus the main obstacle in proving hardness of black-white pebbling is to determine how to modify the construction so that white pebbles will be rendered useless. We exploit an important observation to do this. In 1979, Meyer auf der Heide [7] proved a strong duality between black and white pebbles. Namely, he proved that on any graph $\mathcal{G}$, for any pure black $k$-pebbling strategy there is a pure white $k$-pebbling strategy and vice versa. In order to prove this, he made a modification to the rules of the game. Pure black strategies still begin with an empty graph and end with a single black pebble on the target node, but pure white pebbling strategies now begin with a single pebble on the target node, and end with a completely empty graph. His proof amounts to showing that running a pure black $k$-pebbling strategy backward yields a pure white $k$-pebbling strategy, and vice versa. This has some implications for the original Black-White Pebbling Game, in which every strategy must end with a single black pebble on the target node. Namely, if you try to use as close to a pure white strategy as you can to black pebble the target node of some DAG $\mathcal{G}$ and if the maximum pebbling number $k$ is reached in any pure black strategy of $\mathcal{G}$ at some time when there is no black pebble on the target node, then the black-white strategy will necessarily use $k+1$ pebbles, one black pebble on the target node and $k$ white pebbles which are simulating some optimal black pebbling in reverse. By similar reasoning, if one can build a graph which requires the player to use the maximum number of pebbles in every configuration of every optimal pure black strategy, then using a white pebble in support of a black pebbling of any intermediate node should also exceed the maximum. Our construction is designed to enforce this while maintaining the original properties found in [6].

However, we run into troubles in the case of existentially quantified variables. The problem stems from the fact that for an existential quantifier widget, we want to be able to pebble up to that widget in either of two different ways-one corresponding to the variable being set to true, and the other way corresponding to the variable being set to false. Thus, there is an implicit OR in this argument. This difficulty was also overcome in [6], in the more limited context of black pebbling. If we were constructing monotone circuits rather than graphs (which are special cases of monotone circuits with only AND gates), then things become much easier, even when allowing the use of white pebbles, since we can use an explicit OR gate to allow for either of these two types of pebblings. Our proof of Theorem 10 in Section 3, which uses OR gates as a building block in order to prove an exponential time/space speedup theorem for Resolution, does this and actually implies the PSPACEcompleteness of this problem. However, when OR gates are not allowed, we have to simulate this implicit OR using only AND gates. Any way of doing this will necessarily involve two different pebblings, and it is quite subtle to see how to accomplish this while still prohibiting white pebbles.

\subsection{The Reduction}

To show that the Black-White Pebbling Game is PSPACE-hard, we reduce from QSAT. In our presentation, a QBF $\psi=Q_{n} x_{n} Q_{n-1} x_{n-1} \cdots Q_{1} x_{1} F$, where $F$ is a $3 \mathrm{CNF}$ containing $m$ clauses over the $n$ quantified variables $x_{n}, \ldots, x_{1}$. We have inverted the numbering of the variables simply as a convenience in the proof. Given a QBF $\psi$, we produce a graph $\mathcal{G}$ whose target node $s$ can be black-white pebbled using at most $4 n+3$ pebbles if and only if $\psi$ is in QSAT. Our construction is designed to penalize any use of white pebbles, so that the optimal strategy is all black.

The graph which we construct is composed of $n+$ $m$ widgets, one for each quantified variable and one for each clause in $F$. As in [6], the quantifier widget for $Q_{i} x_{i}$ contains four vertices which represent the variable $x_{i}$, we call these nodes $x_{i}, x_{i}^{\prime}, \bar{x}_{i}, \bar{x}_{i}^{\prime}$. The location of pebbles on these four nodes corresponds to the truth value assigned to $x_{i}$ by the current truth assignment which is being tested by the pebbling. If pebbles are on $x_{i}$ and $\bar{x}_{i}^{\prime}$, then the variable $x_{i}$ is set to true. If pebbles are on $x_{i}^{\prime}$ and $\bar{x}_{i}$ or if pebbles are on $x_{i}^{\prime}$ and $\bar{x}_{i}^{\prime}$, then the variable $x_{i}$ is set to false. Our construction will never allow an assignment to place pebbles on both $x_{i}$ and $\bar{x}_{i}$.

The construction of the quantifier widgets relies on a subwidget we call an $i$-slide. An $i$-slide is designed to severely restrict the player's pebbling strategies. An example of a 4-slide is shown in Figure 1. Once the bottom nodes of an $i$-slide are black-pebbled, the $i$-slide strategy, where the bottom pebbles are slid up to the top nodes in the appropriate order, is the only way to blackpebble the top nodes without exceeding $i$ pebbles.

Definition 2.1: An $i$-slide is a pair of sets $(V, U)$ together with a set of edges that satisfy the following properties. $V$ is a set of $i$ nodes $v^{1}, v^{2}, \cdots, v^{i}$ and $U$ is a set of $i$ nodes $u^{1}, u^{2}, \cdots, u^{i}$ such that: (1) $v^{j}$ is the predecessor of all nodes $v^{k}$ such that $k>j$; (2) $u^{j}$ is the predecessor of all nodes $u^{k}$ such that $k>j$; (3) $u^{j}$ is the predecessor 


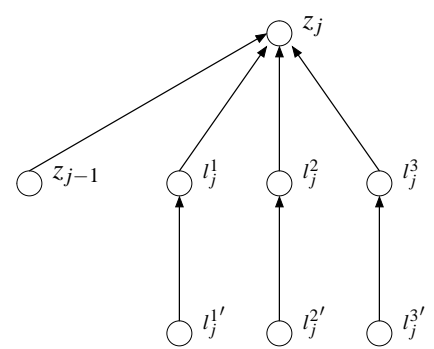

Figure 1. A clause widget for clause $z_{j}=\left(l_{j}^{1} \vee l_{j}^{2} \vee l_{j}^{3}\right)$ (left). The connection of $z_{m}$ to $G_{0}$ (center). And a 4-slide $\left(\left\{v^{1}, v^{2}, v^{3}, v^{4}\right\},\left\{u^{1}, u^{2}, u^{3}, u^{4}\right\}\right)$ (right).

of all nodes $v^{k}$ such that $k \leq j$; (4) $u^{j}$ has at least $i-j+1$ predecessors from outside of $V$ or $U$.

Globally the construction is very much like that in [6]. There are a number of nodes used to encode a truth assignment, which are predecessors to nodes in both clause widgets and quantifier widgets. The clause widgets are connected linearly and can only be pebbled within the space bound of $4 n+3$ if the truth assignment encoded by the current pebbling configuration satisfies $F$. The quantifier widgets are also connected to each other linearly and follow the last clause widget. They slow the advance of the pebbling toward $s$. In order to advance through them, it will be necessary to repebble the clause widgets numerous times, once for each truth assignment required to show that $\psi$ is in QSAT. Only once the final quantifier widget is pebbled is it possible to pebble the target node $s$. We now describe the individual widgets and how they are connected. These descriptions are somewhat terse and are meant to be read in accompaniment to Figures 1, 2, 3, and 4.

The universal widget is depicted in Figure 3 . For every $i, 1 \leq i \leq n$, if widget $i$ is a universal widget, it is composed of 4 groups of nodes, $\left\{\bar{x}_{i}, \bar{x}_{i}^{\prime}, d_{i}, x_{i}, x_{i}^{\prime}, y_{i}\right\}, G_{i-1}=\left\{g_{i-1}^{1}, \ldots, g_{i-1}^{4 i-1}\right\},\left\{a_{i}, b_{i}\right\}$, and $G_{i}=\left\{g_{i}^{1}, \ldots, g_{i}^{4 i+3}\right\}$. These are connected as follows. $y_{i}$ has $4 i+3$ source nodes $p_{x_{i}}^{1}$ through $p_{x_{i}}^{4 i+3}$ as predecessors, $x_{i}^{\prime}$ has $4 i+2$ source nodes $p_{x_{i}}^{1}$ through $p_{x_{i}}^{4 i+2}$ as predecessors, $d_{i}$ has $4 i+1$ source nodes $p_{d_{i}}^{1}$ through $p_{d_{i}}^{4 i+1}$ as predecessors, and $\bar{x}_{i}^{\prime}$ has $4 i$ source nodes $p_{\bar{x}_{i}}^{\frac{1}{x_{i}}}$ through $p_{\bar{x}_{i}}^{4 i}$ as predecessors. The sole predecessor of $x_{i}$ is $x_{i}^{\prime}$ and the sole predecessor of $\bar{x}_{i}$ is $\bar{x}_{i}^{\prime}$. For every pair of nodes $g_{i}^{j}$ and $g_{i}^{k}$ of $G_{i}$, if $j<k$ then $g_{i}^{j}$ is a predecessor of $g_{i}^{k}$. Similarly, for every pair of nodes $g_{i-1}^{j}$ and $g_{i-1}^{k}$ of $G_{i-1}$, if $j<k$ then $g_{i-1}^{j}$ is a predecessor of $g_{i-1}^{k}$. The subgraph $\left(\left\{g_{i}^{1}, \ldots, g_{i}^{4 i-1}\right\}, G_{i-1}\right)$ forms an $4 i-1$ slide. The node $b_{i}$ is a successor of every node in $G_{i-1}$, and the node $a_{i}$ is a successor of every node in $G_{i-1} \cup\left\{b_{i}\right\}$. Finally, $\bar{x}_{i}^{\prime}$ is a predecessor of every node in $\left\{g_{i}^{1}, \ldots, g_{i}^{4 i}\right\}, \bar{x}_{i}$ is a predecessor of $b_{i}, d_{i}$ is a predecessor of both nodes in $\left\{b_{i}, a_{i}\right\}, x_{i}^{\prime}$ is also a predecessor of both nodes in $\left\{b_{i}, a_{i}\right\}$, $x_{i}$ is a predecessor of every node in $\left\{g_{i}^{1}, \ldots, g_{i}^{4 i+1}\right\}, a_{i}$ is a predecessor of every node in $\left\{g_{i}^{1}, \ldots, g_{i}^{4 i+2}\right\}$, and $y_{i}$ is a predecessor of every node in $\left\{g_{i}^{1}, \ldots, g_{i}^{4 i+3}\right\}$.

The existential widget is depicted in Figure 4. For every $i, 1 \leq i \leq n$, if widget $i$ is an existential widget, it is composed of 4 groups of nodes, $\left\{\bar{x}_{i}, \bar{x}_{i}^{\prime}, d_{i}, x_{i}, x_{i}^{\prime}, y_{i}\right\}$, $G_{i-1}=\left\{g_{i-1}^{1}, \ldots, g_{i-1}^{4 i-1}\right\}, R_{i}=\left\{r_{i}^{1}, \ldots, r_{i}^{4 i+1}\right\} \cup H_{i}=$ $\left\{h_{i}^{1}, \ldots, h_{i}^{4 i+1}\right\} \cup\left\{a_{i}\right\}$, and $G_{i}=\left\{g_{i}^{1}, \ldots, g_{i}^{4 i+3}\right\} . x_{i}^{\prime}$ has $4 i+3$ source nodes $p_{x_{i}}^{1}$ through $p_{x_{i}}^{4 i+3}$ as predecessors, $y_{i}$ has $4 i+2$ source nodes $p_{y_{i}}^{1}$ through $p_{y_{i}}^{4 i+2}$ as predecessors $d_{i}$ has $4 i+1$ source nodes $p_{d_{i}}^{1}$ through $p_{d_{i}}^{4 i+1}$ as predecessors, and $\bar{x}_{i}^{\prime}$ has $4 i$ source nodes $p_{\bar{x}_{i}}^{1}$ through $p_{\bar{x}_{i}}^{4 i}$ as predecessors. $\bar{x}_{i}^{\prime}$ also has $y_{i}$ and $x_{i}^{\prime}$ as predecessors. The sole predecessor of $x_{i}$ is $x_{i}^{\prime}$ and the only two predecessors of $\bar{x}_{i}$ are $\bar{x}_{i}^{\prime}$ and $y_{i}$. For every pair of nodes $g_{i}^{j}$ and $g_{i}^{k}$ of $G_{i}$, if $j<k$ then $g_{i}^{j}$ is a predecessor of $g_{i}^{k}$. The same is true for every pair of nodes in $H_{i}, R_{i}$, and $G_{i-1}$. Every node $g_{i}^{j} \in\left\{g_{i}^{1}, \ldots, g_{i}^{4 i+1}\right\}$ has $4 i+1-j$ source nodes as predecessors. Also, $a_{i}$ is a predecessor of every node in $\left\{g_{i}^{1}, \ldots, g_{i}^{4 i+1}\right\}, \bar{x}_{i}^{\prime}$ is a predecessor of every node in $\left\{g_{i}^{1}, \ldots, g_{i}^{4 i+2}\right\}$, and $x_{i}$ is a predecessor of every node in $\left\{g_{i}^{1}, \ldots, g_{i}^{4 i+3}\right\}$. Also, $a_{i}$ is the successor of every node in $H_{i}, d_{i}$ is a predecessor of every node in $\left\{h_{i}^{1}, \ldots, h_{i}^{4 i+1}\right\}$, $\bar{x}_{i}$ is a predecessor of every node in $\left\{h_{i}^{1}, \ldots, h_{i}^{4 i}\right\}$ and $\left(\left\{h_{i}^{1}, \ldots, h_{i}^{4 i-1}\right\}, R_{i}\right)$ forms a $4 i-1$ slide. Finally, $y_{i}$ is a predecessor of every node in $R_{i}$ and $\left(R_{i}, G_{i-1}\right)$ forms a $4 i-1$ slide.

For all $i, 1<i<n, G_{i}$ is part of both widget $i$ and 
widget $i+1 . G_{0}$ is special in that it connects the string of quantifier widgets to the string of clause widgets and is described below. $G_{n}$ is special because every node in $G_{n}$ is a predecessor of the target node $s$. We now describe the $m$ clause widgets.

For each clause $C_{i}$, there is a corresponding node $z_{i}$. This node always has four predecessors, one of which is the previous clause node $z_{i-1}$. The other three, $l_{i}^{1}, l_{i}^{2}$, and $l_{i}^{3}$, correspond to the literals which occur $C_{i}$. For example, if the first literal in the $i^{\text {th }}$ clause is $\bar{x}_{j}$, then the node $\bar{x}_{j}$ from quantifier widget $j$ is one of the predecessors of $z_{i} . z_{1}$ has a special source node $z_{0}$ as a predecessor, since it has no previous clause. Finally, we add edges from $z_{m}$ to all three nodes of $G_{0}$. There are also three source nodes $a_{0}, b_{0}$, and $c_{0}$ which are connected to $G_{0}$. $a_{0}$ and $b_{0}$ are predecessors of $g_{0}^{1}$ and $c_{0}$ is a predecessor of $g_{0}^{2}$. Figure 1 shows both an example of a clause widget as well the connection between $z_{m}$ and $G_{0}$. This completes the construction.

Theorem 2: The QBF $\psi=Q_{n} x_{n} Q_{n-1} x_{n-1} \ldots Q_{1} x_{1} F$ is in QSAT if and only if the target node $s$ of $\mathcal{G}$ can be pebbled with $4 n+3$ pebbles.

Definition 2.2: Let the set of all truth assignments over variables $x_{i+1}, \ldots, x_{n}$ be denoted by $A_{i}$. Thus each $\alpha_{i}$ in $A_{i}$ is a partial assignment that sets the outermost $n-i$ variables of $Q_{n} x_{n} \ldots Q_{1} x_{1} F$. We use the notation $\left(v_{1}, v_{2}\right)$ to denote $v_{1}$ or $v_{2}$. For any assignment to $\alpha_{i}$, define $B_{\alpha_{i}}$ to be the pebbling configuration of $\mathcal{G}$ consisting of black pebbles on the following nodes: For each universally quantified variable $x_{j}$ of $\psi, j \geq i+1$, if $\alpha_{i}\left(x_{j}\right)=0$, then $y_{j} \in B_{\alpha_{i}}, x_{j}^{\prime} \in B_{\alpha_{i}}, d_{j} \in B_{\alpha_{i}}$, and $\left(\bar{x}_{j}, \bar{x}_{j}^{\prime}\right) \in B_{\alpha_{i}}$. Otherwise, if $\alpha_{i}\left(x_{j}\right)=1$, then $y_{j} \in B_{\alpha_{i}}, \bar{x}_{j}^{\prime} \in B_{\alpha_{i}}, a_{j} \in B_{\alpha_{i}}$ and $\left(x_{j}, x_{j}^{\prime}\right) \in B_{\alpha_{i}}$. For each existentially quantified variable $x_{j}$ of $\psi, j \geq i+1$, if $\alpha_{i}\left(x_{j}\right)=0$, then $y_{j} \in B_{\alpha_{i}}, x_{j}^{\prime} \in B_{\alpha_{i}}$, $d_{j} \in B_{\alpha_{i}}$, and $\left(\bar{x}_{j}, \bar{x}_{j}^{\prime}\right) \in B_{\alpha_{i}}$. Otherwise, if $\alpha_{i}\left(x_{j}\right)=1$, then $y_{j} \in B_{\alpha_{i}}, \bar{x}_{j}^{\prime} \in B_{\alpha_{i}}, d_{j} \in B_{\alpha_{i}}$ and $\left(x_{j}, x_{j}^{\prime}\right) \in B_{\alpha_{i}}$.

Definition 2.3 (Black clamping interval) Let $t_{0} \leq t_{j} \leq$ $t_{k} \leq t_{\text {end }}$. For any node $v$, we say $v \in\left[t_{a}, t_{b}\right]$ if $v$ is black pebbled during every configuration from time $t_{a}$ through time $t_{b}$. More generally, for any pair of nodes $(u, v)$, we say that $(u, v) \in\left[t_{a}, t_{b}\right]$ if either $u$ or $v$ is black pebbled during every configuration from time $t_{a}$ to time $t_{b}$. Let $S$ be a set of pairs of nodes. We say that $S \subseteq\left[t_{a}, t_{b}\right]$ if for all $(u, v) \in S,(u, v) \in\left[t_{a}, t_{b}\right]$. Note that $u$ and $v$ may be the same nodes.

Lemma 3: If $\psi$ is in QSAT, then the target node $s$ of $\mathcal{G}$ can be pebbled with $4 n+3$ pebbles.

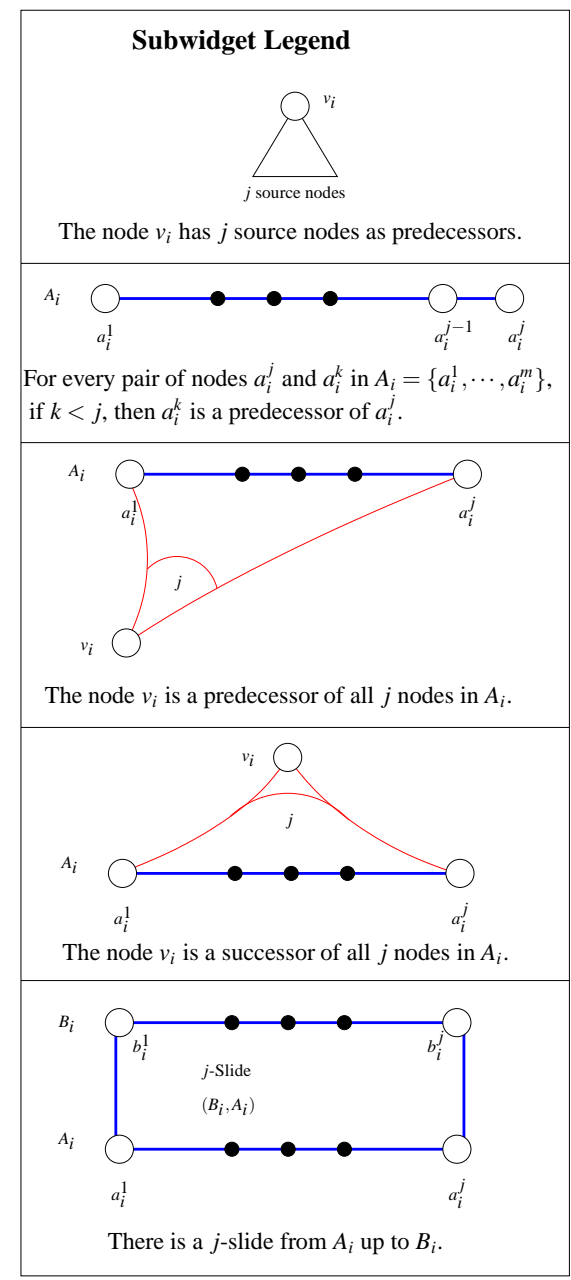

\section{Figure 2. Legend explaining the compo- nents of Figures 3 and 4.}

Lemma 11 follows from the following more general lemma by setting $i=n$.

Lemma 4: For all $i, \alpha_{i} \in A_{i}$, suppose the graph $\mathcal{G}$ is initially in configuration $B_{\alpha_{i}}$. If $\psi$ is in QSAT, then we can black pebble $G_{i}$ at some time $t>1$ using $4 n+3$ pebbles, while keeping $B_{\alpha_{i}}$ clamped (i.e., $B_{\alpha_{i}} \in[1, t]$.)

Proof: The proof is by induction on $i$ from 0 to $n$. The base case is when $i=0$. Let $\alpha_{0}$ be any assignment in $A_{0}$. Suppose that $Q_{n} x_{n} \cdots Q_{1} x_{1} F\left\lceil\alpha_{0}\right.$ is in QSAT. Then some literal in every clause must be set to true. This implies that for each $z_{j}, 1 \leq j \leq m$, at least one of $l_{j}^{1}, l_{j}^{2}$, or $l_{j}^{3}$ are black pebbled in $B_{\alpha_{0}}$. We can therefore black pebble $G_{0}$ as follows. Start by putting a black pebble 


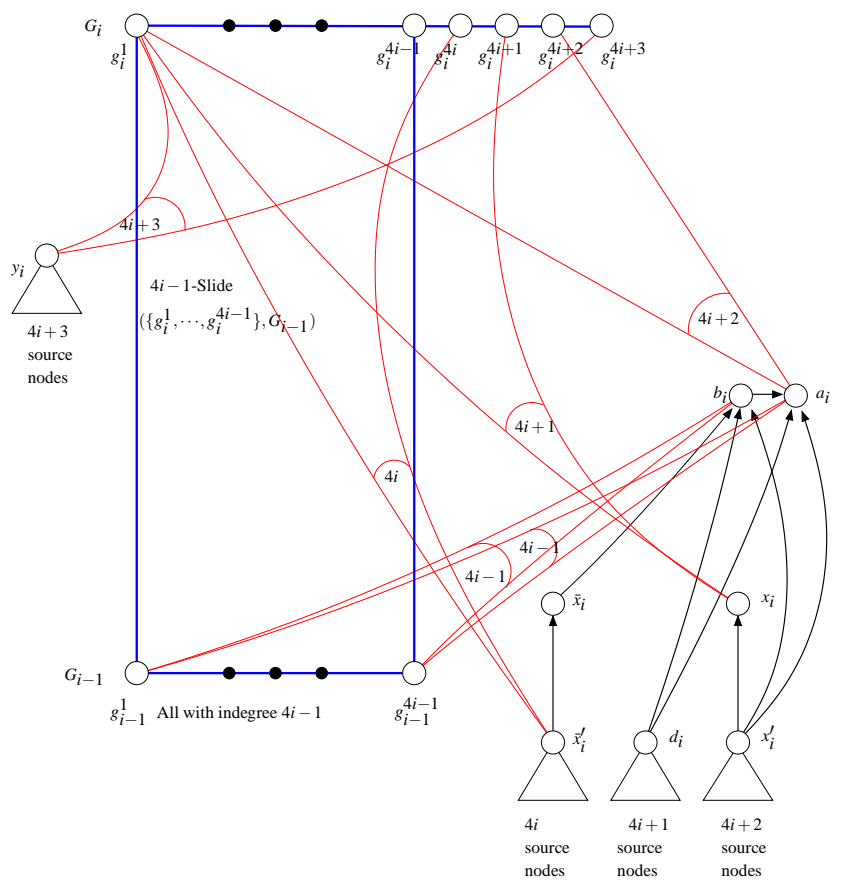

Figure 3. A universal widget.

on $z_{0}$. Then since at most two of $z_{1}$ 's other predecessors are unpebbled, we have enough free pebbles to black pebble the rest of $z_{1}$ 's predecessors. We know we can black pebble them because if some $l_{1}^{k}$ is unpebbled, then $l_{1}^{k^{\prime}}$ must be black pebbled in $B_{\alpha_{i}}$. We can therefore black pebble all of $z_{1}$ 's predecessors. We can then slide the pebble from $z_{0}$ to $z_{1}$ and lift the other (at most 2) pebbles which we just put down. Once $z_{1}$ is black pebbled, we can then black pebble $z_{2}$ the same way, all the way to $z_{m}$. Once $z_{m}$ is black pebbled we can use the remaining two black pebbles to black pebble $a_{0}$ and $b_{0}$, and then slide the pebble from $z_{m}$ to $c_{0}$. We can then slide the black pebble from $a_{0}$ to $g_{0}^{1}$, from $b_{0}$ to $g_{0}^{2}$, and from $c_{0}$ to $g_{0}^{3}$. Note that this strategy uses only black pebbles. For the inductive step there are two cases depending on whether $Q_{i}$ is a universal or an existential quantifier.

Case 1: $Q_{i}$ is a universal quantifier. In this case, both $\psi\left\lceil\alpha_{i} \cup\left\{x_{i}\right\}\right.$ and $\psi\left\lceil\alpha_{i} \cup\left\{\bar{x}_{i}\right\}\right.$ are in QSAT. We begin in configuration $B_{\alpha_{i}}$ with $4 i+3$ free pebbles. Black pebble $y_{i}$, followed by $x_{i}^{\prime}$, then $d_{i}$, and then $\bar{x}_{i}^{\prime}$. Then move the pebble from $\bar{x}_{i}^{\prime}$ to $\bar{x}_{i}$. At this point we have $4 i-1$ pebbles free and can apply the induction hypothesis to black pebble $G_{i-1}$. Then slide the black pebble from $\bar{x}_{i}$ to $b_{i}$, then the black pebble from $d_{i}$ to $a_{i}$. Remove all pebbles from widget $i$ except for the ones on $a_{i}, x_{i}^{\prime}$, and $y_{i}$. Then slide the black pebble from $x_{i}^{\prime}$ to $x_{i}$ and black pebble $\bar{x}_{i}^{\prime}$ again. Now apply the induction hypothesis to simultaneously black pebble $G_{i-1}$ again. Next, use the $i$-slide strategy to slide all of $G_{i-1}$ 's pebbles to $\left\{g_{i}^{1}, \ldots, g_{i}^{4 i-1}\right\}$. Then slide $\bar{x}_{i}^{\prime}$ 's black pebble to $g_{i}^{4 i}$, and then $x_{i}$ 's black pebble to $g_{i}^{4 l+1}$. Next slide the black pebble from $a_{i}$ to $g_{i}^{4 i+2}$ Finally, slide the black pebble from $y_{i}$ to $g_{i}^{4 i+3}$.

Case 2: $Q_{i}$ is an existential quantifier. In this case, either $\psi\left\lceil\alpha_{i} \cup\left\{x_{i}\right\}\right.$ or $\psi\left\lceil\alpha_{i} \cup\left\{\bar{x}_{i}\right\}\right.$ is in QSAT. As in the universal case, we begin in $B_{\alpha_{i}}$ with $4 i+3$ free pebbles. Black pebble $x_{i}^{\prime}$, followed by $y_{i}, d_{i}$, and then $\bar{x}_{i}{ }^{\prime}$.

If $\psi\left\lceil\alpha_{i} \cup\left\{x_{i}\right\}\right.$ is in QSAT, move the black pebble from $x_{i}^{\prime}$ to $x_{i}$. Then apply the induction hypothesis to black pebble $G_{i-1}$. Then use the $i$-slide strategy to move all of the pebbles from $G_{i-1}$ to $R_{i}$. Then slide the black pebble from $y_{i}$ to $\bar{x}_{i}$. Then use the $i$-slide strategy to move all of the pebbles from $R_{i}$ to $\left\{h_{i}^{1}, \ldots, h_{i}^{4 i-1}\right\}$. After that, slide the pebble from $\bar{x}_{i}$ to $h_{i}^{4 i}$ and then slide the pebble from $d_{i}$ to $h_{i}^{4 i+1}$. Then slide the pebble from $h_{i}^{4 i+1}$ to $a_{i}$. At this point remove all the pebbles off of the widget so that only $\bar{x}_{i}^{\prime}, x_{i}$, and $a_{i}$ remain. Use the $4 i$ free pebbles to pebble the source node predecessors of $g_{i}^{1}$ and then slide one to $g_{i}^{1}$ itself. Use the pebbles left over on the source nodes to subsequently pebble each $g_{i}^{j}$ until $g_{i}^{4 i}$ is pebbled. At this point slide the pebble from $a_{i}$ to $g_{i}^{4 i+1}$, slide the pebble from $\bar{x}_{i}^{\prime}$ to $g_{i}^{4 i+2}$, and finish by sliding the pebble from $x_{i}$ to $g_{i}^{4 i+1}$.

If $\psi \Gamma_{\alpha_{i} \cup\left\{\bar{x}_{i}\right\}}$ is in QSAT, move the black pebble from $\bar{x}_{i}^{\prime}$ to $\bar{x}_{i}$. Then apply the induction hypothesis to black pebble $G_{i-1}$. Then use the $i$-slide strategy to move all of the pebbles from $G_{i-1}$ to $R_{i}$. Then use the $i$-slide strategy to move all of the pebbles from $R_{i}$ to $\left\{h_{i}^{1}, \ldots, h_{i}^{4 i-1}\right\}$. After that, slide the pebble from $\bar{x}_{i}$ to $h_{i}^{4 i}$ and then slide the pebble from $d_{i}$ to $h_{i}^{4 i+1}$. Then slide the pebble from $h_{i}^{4 i+1}$ to $a_{i}$. At this point remove all the pebbles off of the widget so that only $y_{i}, x_{i}^{\prime}$, and $a_{i}$ remain. Use the $4 i$ pebbles that are free to repebble $\bar{x}_{i}^{\prime}$ and then pick the pebble up from $y_{i}$ and pick up the $4 i-1$ pebbles that remain on the source node predecessors of $\bar{x}_{i}^{\prime}$. Slide the pebble from $x_{i}^{\prime}$ to $x_{i}$. At this point $\bar{x}_{i}^{\prime}, x_{i}$, and $a_{i}$ are all pebbled and we can finish by black pebbling $G_{i}$ as we did in the positive case.

Lemma 5: Let $\psi$ be a QBF, and let $\mathcal{G}$ be the corresponding graph. If $s$ has a $4 n+3$ black-white pebbling strategy in $\mathcal{G}$, then $\psi$ is in QSAT, and any $4 n+3$ blackwhite pebbling strategy requires $\Omega\left(2^{k}\right)$ steps, where $k$ is the number of universal quantifiers in $\psi$.

We first note that $s$ has $4 n+3$ predecessors, $G_{n}$. Each of these nodes has indegree $4 n+3$. So no node of $G_{n}$ could ever contain a white pebble while $s$ contains a 


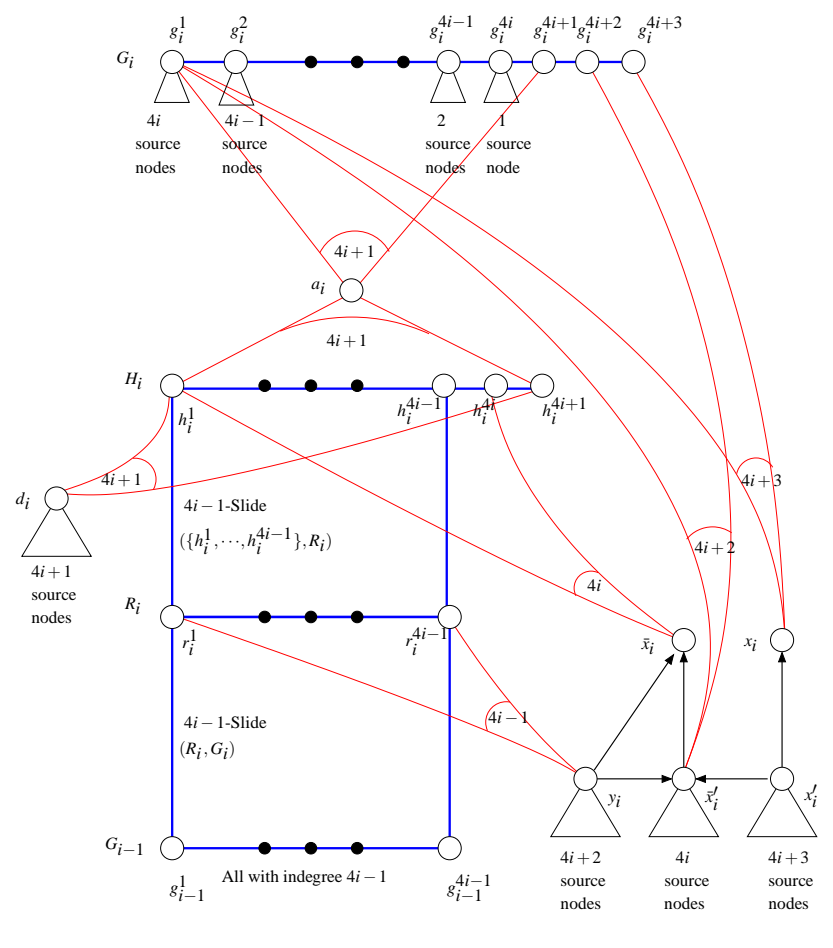

Figure 4. An existential widget.

black pebble, because there would not be enough free pebbles to discharge it. Therefore, in order to pebble $s$, $G_{n}$ must first be simultaneously black pebbled. Lemma 5 therefore follows from the more general Lemma 6.

Lemma 6: For all $\alpha_{i} \in A_{i}$, if there exists times $t^{\prime}, t^{\prime \prime}$ such that $B_{\alpha_{i}} \subseteq\left[t^{\prime}, t^{\prime \prime}\right]$, then black pebbling $G_{i}$ at $t^{\prime \prime}$ from $B_{\alpha_{i}}$ using no more than $4 n+3$ pebbles, requires that $\psi$ is in QSAT and requires $\Omega\left(2^{k}\right)$ units of time between $t^{\prime}$ and $t^{\prime \prime}$, where $k$ is the number of universal quantifiers among the $i$ inner most quantifiers.

The following lemma will be used repeatedly. In particular, it implies that for any $i$-slide $(V, U)$, in order to pebble $V$ using no more than $i$ pebbles, $U$ must first be black pebbled at some earlier time.

Lemma 7: Let $\mathcal{G}$ be a DAG which is to be $k$ pebbled. Let $v$ be a node of $\mathcal{G}$ which has $c$ predecessors $p_{1}, \ldots, p_{c}$. Let $U$ be a set of $k-c$ pairs of nodes such that: 1) $\left.U \subseteq\left[t^{\prime}, t^{\prime \prime}\right], 2\right)$ for each $\left(x_{1}, x_{2}\right) \in U$, neither $x_{1}$ nor $x_{2}$ in $\left.\left\{v, p_{1}, \ldots, p_{c}\right\}, 3\right)$ for each $\left(x_{1}, x_{2}\right) \in U$ where $x_{1} \neq x_{2}$, neither $x_{1}$ nor $x_{2}$ is a descendant of $v$. Finally, suppose $v$ is not white pebbled at $t^{\prime \prime}$. Then between $t^{\prime}$ and $t^{\prime \prime}, v$ can be black pebbled at most once and cannot be white pebbled.
Proof: Suppose $v$ is white pebbled at some time between $t^{\prime}$ and $t^{\prime \prime}$. Then, by frugality, its white pebble can only be discharged once it has contributed toward placing a black pebble on some descendant $z$ of $v$. Since $\mathcal{G}$ is acyclic, $z$ cannot be a predecessor of $v$. Also, since all of the nodes in $U$ either have their black pebbles fixed between $t^{\prime}$ and $t^{\prime \prime}$ or are not descendants of $v, z$ cannot be in $U$. So there are $k-c$ black pebbles in $U, 1$ black pebble on $z$, and 1 white pebble on $v$. This means that at most $c-2$ pebbles can be placed on $v$ 's predecessors without exceeding the limit of $k$. Therefore, it is impossible to discharge $v$ 's white pebble until after $t^{\prime \prime}$. But since $v$ is not white pebbled at $t^{\prime \prime}, v$ cannot be white pebbled in the first place. A similar argument forbids a second black pebbling.

Proof: [of Lemma 6] The proof is by induction on $i$ from 0 to $n$. The base case is when $i=0$. Let $\alpha_{0}$ be any assignment in $A_{0}$ and suppose there exist times $t^{\prime}$ and $t^{\prime \prime}$ such that $B_{\alpha_{0}} \subseteq\left[t^{\prime}, t^{\prime \prime}\right]$. We will show that simultaneously black pebbling $G_{0}$ at $t^{\prime \prime}$ without ever exceeding $4 n+3$ pebbles requires that $\psi$ is in QSAT.

In order to black pebble $z_{j}$ or discharge a white pebble from $z_{j}$ we must either black pebble $z_{j-1}$ or discharge a white pebble from $z_{j-1}$. In order to black pebble any node in $G_{0}$, we must pebble $z_{m}$. Inductively, this means that at some point for every single $z_{j}$, it was necessary to either black pebble it or discharge a white pebble from it. But every $z_{j}$ (except $z_{0}$ ) has 4 predecessors, $l_{j}^{1}, l_{j}^{2}, l_{j}^{3}, z_{j-1}$. Therefore, in order to pebble $z_{j}$ at least one $l_{j}^{k}$ must be black pebbled in $B_{\alpha_{0}}$. But in this case, $\alpha_{0}$ must satisfy clause $j$ of $F$. Since every $z_{j}$ must either be black pebbled or discharged, $\alpha_{0}$ must satisfy every clause of $F$. Therefore $F\left\lceil\alpha_{0}\right.$ is in QSAT.

Induction Step: We now prove the induction step in which we will show that if we can simultaneously black pebble $G_{i}=\left\{g_{i}^{1} \cdots g_{i}^{4 i+3}\right\}$ using no more than $4 i+3$ pebbles without moving any pebbles in $B_{\alpha_{i}}$, then $\psi\left\lceil\alpha_{i}\right.$ is in QSAT and the pebbling must take time $\Omega\left(2^{k}\right)$, where $k$ is the number of universally quantified variables among the inner most $i$ variables of $\psi$.

Case 1: $Q_{i}$ is a universal quantifier. We will show that in order to black pebble $G_{i}$ we must necessarily pass through a number of all-black configurations, including black pebbling $G_{i-1}$ twice, once with black pebbles on $x_{i}^{\prime}, d_{i}$, and either $\bar{x}_{i}$ or $\bar{x}_{i}^{\prime}$ (the false configuration), and once with black pebbles on $\bar{x}_{i}^{\prime}, a_{i}$, and either $x_{i}$ or $x_{i}^{\prime}$ (the true configuration).

We appeal to Lemma 7 to conclude that since $y_{i}$ has $4 i+3$ source nodes as predecessors, our first action within widget $i$ must be to black pebble $y_{i}$ and it must stay in place until its last successor $g_{i}^{4 i+3}$ is pebbled for 
the final time at $t_{15}$, so $y_{i} \in\left[t_{1}, t_{15}-1\right]$.

Now that $y_{i}$ is clamped, we can appeal to Lemma 7 to conclude that no node in $G_{i} \cup\left\{a_{i}, b_{i}, x_{i}^{\prime}\right\}$ can be white pebbled and each can only be black pebbled once between $t_{1}$ and $t_{15-1}$. Since $x_{i}^{\prime}$ has $4 i+2$ source nodes as predecessors, our second action within widget $i$ must be to black pebble $x_{i}^{\prime}$ and it must stay in place until its successor $x_{i}$ is pebbled for the last time. Then a pebble must remain on $x_{i}$ until all of its successors are pebbled for the last time, because we can never repebble/discharge $x_{i}$ once $x_{i}^{\prime}$ is empty. Let $t_{7}$ be the time that $a_{i}$ is pebbled and let $t_{12}$ be the time $g_{i}^{3 i}$ is pebbled. Then $x_{i}^{\prime} \in\left[t_{2}, t_{7}-1\right]$ and $\left(x_{i}, x_{i}^{\prime}\right) \in\left[t_{7}, t_{12}-1\right]$.

Our argument now divides into two sections. In order to simultaneously black pebble $G_{i}$ we must black pebble $g_{i}^{4 i+3}$, which requires that both $a_{i}$ and $\left\{g_{i}^{1}, \ldots, g_{i}^{4 i}\right\}$ be pebbled. In the first part of the argument we prove that in order to black pebble $a_{i}, \psi\left\lceil_{\alpha_{i} \cup\left\{\bar{x}_{i}\right\}}\right.$ must be QSAT and that $\Omega\left(2^{k}\right)$ units of time must pass between $t_{0}$ and $t_{7}$, where $k$ is the number of universally quantified variables among the inner most $i-1$ variables of $\psi$. In the second part of the argument, we argue that $g_{i}^{1}, \ldots, g_{i}^{4 i}$ must also be simultaneously black pebbled in order to black pebble $g_{i}^{4 i+3}$ and that pebbling them without exceeding our bound necessitates that $\psi\left\lceil\alpha_{i} \cup\left\{x_{i}\right\}\right.$ is in QSAT and that $\Omega\left(2^{k}\right)$ units of time pass between times $t_{7}$ and $t_{14}-1$. This will allow us to conclude that black pebbling $G_{i}$ requires that $\psi\left\lceil\alpha_{i}\right.$ is in QSAT and requires $\Omega\left(2^{k^{\prime}}\right)$ time, where $k^{\prime}=k+1$ is the number of universally quantified variables among the inner most $i$ variables of $\psi$.

Since $a_{i}$ can only be black pebbled once and is needed to pebble each node of $G_{i}, a_{i} \in\left[t_{7}, t_{14}-1\right]$. In order to black pebble $a_{i}$ at time $t_{7}$ we must pebble $b_{i}$ at some time $t_{6}$, before $t_{7}$. Again, we know that $b_{i}$ can only be black pebbled once in $t_{1}$ to $t_{14}$, so $b_{i} \in\left[t_{6}, t_{7}-1\right]$. Also, $d_{i}$ is a predecessor of both $a_{i}$ and $b_{i}$ and must be pebbled at times $t_{6}-1$ and $t_{7}-1$. Since $x_{i}^{\prime}$ is in $\left[t_{2}, t_{7}\right]$, by Lemma 7 we can conclude that $d_{i}$ cannot be white pebbled and can only be black pebbled once in this interval. Also, since it has in-degree $4 i, d_{i}$ must be black pebbled at $t_{3}$, immediately after $t_{2}$ as in Lemma 11 , so $d_{i} \in\left[t_{3}, t_{7}-1\right]$. The same argument can be made to argue that $\left(\bar{x}_{i}, \bar{x}_{i}^{\prime}\right) \in$ $\left[t_{4}, t_{6}-1\right]$, where $t_{4}$ is after $t_{3}$. In order to black pebble $a_{i}$ or $b_{i}$, we must first pebble $G_{i-1}$ at some time $t_{5}$ before $t_{6}$. This whole time the nodes $x_{i}^{\prime}, d_{i}$, and $\left(\bar{x}_{i}, \bar{x}_{i}^{\prime}\right)$ are clamped. We can therefore apply Lemma 7 to conclude that $G_{i-1}$ must be black pebbled at some time $t_{5}$ between $t_{4}$ and $t_{6}$. We can now apply the induction hypothesis to conclude that black pebbling $G_{i-1}$ requires $\psi\left\lceil_{\alpha_{i} \cup\left\{\bar{x}_{i}\right\}}\right.$ to be QSAT and black pebbling $G_{i-1}$ from $B\left[t_{4}\right]$ requires time $\Omega\left(2^{k}\right)$, where $k$ is the number of universally quantified variables among the inner most $i-1$ variables of $\psi$.

We now proceed with the second phase of the argument. We know that each node in $G_{i}$ cannot be white pebbled and can only be black pebbled once. So when we black pebble $g_{i}^{4 i+3}$ at time $t_{15}$, all the rest of $G_{i}$ must already be black pebbled. Consider $g_{i}^{4 i+2}$. In order to black pebble it at time $t_{14}$ before $t_{15}$, we must first black pebble $g_{i}^{4 i+1}$ at time $t_{13}$ before $t_{14}$. In order to black pebble $g_{i}^{4 i+1}$ at time $t_{13}$ we must first black pebble $g_{i}^{4 i}$ at time $t_{12}$ and in order to pebble that, we must pebble $g_{i}^{1}, \ldots, g_{i}^{4 i-1}$ at time $t_{11}$. But we must also pebble $\bar{x}_{i}^{\prime}$. Note that $\bar{x}_{i}^{\prime}$ must be empty at $t_{7}$ since $y_{i}$ is clamped and $a_{i}$ has $4 i+2$ other predecessors, none of which is $\bar{x}_{i}^{\prime}$. Also, $\bar{x}_{i}^{\prime}$ must be empty again by $t_{13}-1$, since $g_{i}^{4 i+1}$ has $4 i+3$ predecessors, none of which is $\bar{x}_{i}^{\prime}$. We can therefore apply Lemma 7 to conclude that between $t_{7}$ and $t_{13}$, $\bar{x}_{i}^{\prime}$ cannot be white pebbled and can only be black pebbled once in that interval. We must therefore repebble $\bar{x}_{i}^{\prime}$ at some time $t_{8}$ after $t_{7}$ when $a_{i}$ and $\left(x_{i}, x_{i}^{\prime}\right)$ are clamped and $\bar{x}_{i}^{\prime} \in\left[t_{8}, t_{12}-1\right]$. Since $\bar{x}_{i}^{\prime}$ is a predecessor of every node in $g_{i}^{1}, \ldots, g_{i}^{4 i-1}$, these nodes can only be black pebbled at some time $t_{11}$, with $g_{i}^{1}$ being pebbled first at $t_{10}$, after $t_{8}$. Every node of $G_{i-1}$ is a predecessor of $g_{i}^{1}$. Since the three nodes $\left\{\bar{x}_{i}^{\prime}, a_{i},\left(x_{i}, x_{i}^{\prime}\right)\right\}$ are clamped during the interval $\left[t_{7}, t_{11}\right]$ we can apply Lemma 7 to conclude that $G_{i-1}$ must be black pebbled at $t_{9}$ between $t_{8}$ and $t_{10}$. Since $\left\{\bar{x}_{i}^{\prime}, a_{i},\left(x_{i}, x_{i}^{\prime}\right)\right\}$ is the true assignment for variable $x_{i}$ we can apply our induction hypothesis to conclude that $\psi\left\lceil_{\alpha_{i} \cup\left\{x_{i}\right\}}\right.$ must be QSAT and black pebbling $G_{i-1}$ from $B\left[t_{7}\right]$ requires time $\Omega\left(2^{k}\right)$, where $k$ is the number of universally quantified variables among the inner most $i-1$ variables of $\psi$.

Thus we have shown that any $4 n+3$ pebbling must black pebble $G_{i-1}$ twice between $t_{0}$ and $t_{15}$, once implying that $\psi\left\lceil_{\alpha_{i} \cup\left\{\bar{x}_{i}\right\}}\right.$ is in QSAT, and once implying that $\psi\left\lceil\alpha_{i} \cup\left\{x_{i}\right\}\right.$ is in QSAT. Each time requires $\Omega\left(2^{k}\right)$ time, where $k$ is the number of universally quantified variables among the inner most $i-1$ variables of $\psi$. Therefore, black pebbling $G_{i}$ requires time $\Omega\left(2^{k+1}\right)$, and implies that $\psi\left\lceil_{\alpha_{i}}\right.$ is in QSAT.

Case 2: $Q_{i}$ is an existential quantifier. We will show that in order to black pebble $G_{i}$, we must necessarily pass through a number of all-black partial configurations, including simultaneously black pebbling $G_{i-1}$, either with black pebbles on $x_{i}^{\prime}, d_{i}$, and either $\bar{x}_{i}$ or $\bar{x}_{i}^{\prime}$ (the false configuration), or with black pebbles on $\bar{x}_{i}^{\prime}, d_{i}$, and either $x_{i}$ or $x_{i}^{\prime}$ (the true configuration).

By Lemma 7, no node in $G_{i} \cup\left\{x_{i}^{\prime}\right\}$ can be white pebbled between $t_{0}$ and $t_{15}$, and each can be black pebbled at most once. Based on which nodes of $G_{i}$ are predecessors to others, we can conclude that $g_{i}^{4 i+3}$ must be black 
pebbled last, at time $t_{15}, g_{i}^{4 i+2}$ must be black pebbled before that at time $t_{14}$ and $g_{i}^{4 i+2} \in\left[t_{14}, t_{15}\right]$, and $g_{i}^{4 i+1}$ must be pebbled before that at time $t_{13}$ and $g_{i}^{4 i+1} \in\left[t_{13}, t_{15}\right], g_{i}^{1}$ must be pebbled before that at time $t_{12}$ and $g_{i}^{1} \in\left[t_{12}, t_{15}\right]$. Also, $\left(x_{i}, x_{i}^{\prime}\right) \in\left[t_{1}, t_{15}-1\right]$.

Now consider $y_{i}$. It has degree $4 i+2$, and it must be black pebbled at time $t_{2}$, and can never be repebbled again. Thus it must remain black pebbled until it is used for the last time.

Clearly, both $\bar{x}_{i}^{\prime} \in B\left[t_{12}-1\right]$ and $a_{i} \in B\left[t_{12}-1\right]$. Let $t_{11}$ be the last time $a_{i}$ is pebbled. At this time, $a_{i}$ must be pebbled black. We can see this because $g_{i}^{4 i+1}$ cannot get its black pebble from $g_{i}^{1}$ through to $g_{i}^{4 i}$ since these can only be pebbled once. All of these must be in place when $g_{i}^{4 i+1}$ gets its black pebble, so it cannot get a black pebble from either $x_{i}$ or $\bar{x}_{i}^{\prime}$ since both of these are needed to support $g_{i}^{4 i+2}$ and could not be repebbled with so many black pebbles clamped in $G_{i} . g_{i}^{4 i+1}$,s $4 i+3^{r d}$ predecessor is $a_{i}$, so it must receive its black pebble via a slide move from $a_{i}$. So $a_{i}$ must be black during the interval $\left[t_{11}, t_{12}-1\right]$

At this point our proof splits into two cases, either a black pebble is on $\bar{x}_{i}^{\prime}$ at $t_{11}$ or not. One of these cases will imply that $\psi\left\lceil\alpha_{i} \cup\left\{x_{i}\right\}\right.$ is in QSAT and the other one will imply that $\psi\left\lceil_{\alpha_{i} \cup\left\{\bar{x}_{i}\right\}}\right.$ is in QSAT.

Suppose there is no black pebble on $\bar{x}_{i}^{\prime}$ at $t_{11}$. Then there are two subcases to consider. Subcase (i): there is no pebble at all on $\bar{x}_{i}^{\prime}$ or subcase (ii) there is a white pebble on $\bar{x}_{i}^{\prime}$ at $t_{11}$. First we consider subcase (i): there is no pebble at all on $\bar{x}_{i}^{\prime}$. Then we must repebble $\bar{x}_{i}^{\prime}$ at some time $t^{*}$ between $t_{11}$ and $t_{12-1}$. We will first argue that two nodes, $x_{i}^{\prime}$ and $y_{i}$ must be clamped during the interval $\left[t_{2}, t_{11}\right]$. First, because $x_{i}^{\prime}$ is black pebbled at $t_{1}$, and is a predecessor of $\bar{x}_{i}^{\prime}$, and can never be pebbled again (because its indegree is $4 i+3$ ), it follows that $x_{i}^{\prime} \in\left[t_{1}, t^{*}-1\right]$. Secondly, since $y_{i}$ is a predecessor of $\bar{x}_{i}^{\prime}$ (and by the above reasoning gets black pebbled only once at $\left.t_{2}\right)$, it follows that $y_{i} \in\left[t_{2}, t^{*}-1\right]$. Thus both $x_{i}^{\prime}$ and $y_{i}$ are clamped during the interval $\left[t_{2}, t_{11}\right]$.

Now we will argue that each node of $H_{i}$ must be black pebbled, and can only be pebbled once. Let $t_{10}$ be the time when $h_{i}^{4 i+1}$ is pebbled; let $t_{9}$ be the time when $h_{i}^{4 i}$ is pebbled; let $t_{8}$ be the time when $h_{i}^{4 i-1}$ is pebbled, and let $t_{7}$ be the time when $h_{i}^{1}$ is pebbled, where $t_{7}<t_{8}<t_{9}<$ $t_{10}$. By Lemma 7 and because $x_{i}^{\prime}$ and $y_{i}$ are clamped, and all nodes in $H_{i}$ have indegree $4 i+1$, it follows that each can only be pebbled once and must be pebbled black. Thus, $h_{i}^{4 i+3} \in\left[t_{10}, t_{11}-1\right], h_{i}^{4 i+2} \in\left[t_{9}, t_{11}-1\right], h_{i}^{4 i+1} \in$ $\left[t_{8}, t_{11}-1\right]$, and $h_{i}^{1} \in\left[t_{7}, t_{11}-1\right]$.

Next we will argue that during the interval $\left[t_{3}, t_{10}-1\right]$, the three nodes $d_{i}, x_{i}^{\prime}$ and $y_{i}$ are all black clamped. (We already know that $x_{i}^{\prime}$ and $y_{i}$ are black clamped during this interval.) Because $d_{i}$ has indegree $4 i+1$, by Lemma 7 , again we know that $d_{i}$ must be black pebbled at time $t_{3}$ and can only be black pebbled once. Thus, $d_{i}$ is black and clamped during the interval $\left[t_{3}, t_{10}-1\right]$.

Now again we can apply Lemma 7 to $R_{i}$. Since 3 of the widget's nodes are clamped during this interval, and since all nodes in $R_{i}$ have degree $4 i$, it follows that they can only be pebbled once between $t_{3}$ and $t_{10}-1$ and are black. Let $t_{6}$ be the time $r_{i}^{1}$ is pebbled, $t_{6}<t_{7}$.

Finally, we want to show that $\left(\bar{x}_{i}, \bar{x}_{i}^{\prime}\right) \in\left[t_{3}+1, t_{9}-\right.$ 1] and furthermore the pebbled node is black. First, $\bar{x}_{i}$ must be pebbled at time $t_{7}-1$ because it is a predecessor of $h_{i}^{1}$. Furthermore we will argue that it must be black pebbled. At time $t_{7}-1, \bar{x}_{i}^{\prime}$ must be unpebbled because in order to pebble $h_{i}^{1}$ at time $t_{7}$, there must be $4 i+3$ pebbles already on this $i^{\text {th }}$ widget, not including $\bar{x}_{i}^{\prime}$ (the $4 i+1$ predecessors of $h_{i}^{1}$ plus the clamped nodes $x_{i}^{\prime}$ and $y_{i}$.) Similarly, $\bar{x}_{i}^{\prime}$ must unpebbled at $t_{9}$. Now if $\bar{x}_{i}$ were pebbled white rather than black at $t_{7}-1$, it would have to be discharged by $t_{9}$; but this cannot happen since it would have to be discharged through the unpebbled $\bar{x}_{i}^{\prime}$, which would exceed our allowable space. Thus we have argued that $\bar{x}_{i}$ must be pebbled black at $t_{7}-1$, and further remains black until $t_{9}-1$ since it is a predecessor of each node in $\left\{h_{i}^{1}, \ldots, h_{i}^{4 i}\right\}$.

Now to black pebble $\bar{x}_{i}$ by $t_{7}-1, \bar{x}_{i}^{\prime}$ must be pebbled earlier, say at time $t_{4}, t_{3}<t_{4}<t_{7}-1$. It is left to argue that $t_{4}=t_{3}+1$. When we black pebble $\bar{x}_{i}^{\prime}$ at time $t_{4}$, we have already argued that there are three nodes already clamped, $x_{i}^{\prime}, y_{i}$ and $d_{i}$. Because $\bar{x}_{i}^{\prime}$ has indegree $4 i$, it follows that it must be black pebbled next, and can only be pebbled once. Thus $\left(\bar{x}_{i}, \bar{x}_{i}^{\prime}\right) \in\left[t_{3}+1, t_{9}-1\right]$.

Now in order to black pebble $r_{i}^{1}$ at $t_{6}$, every node of $G_{i-1}$ must be pebbled at $t_{6}-1$. Again we can apply Lemma 7. Since there are 4 nodes clamped, and the degree of each node in $G_{i}$ is $4 i-1$, it follows by our lemma that every node in $G_{i}$ can only be pebbled once between $t_{4}$ and $t_{9}$ and must be black pebbled. Now finally we can apply the induction hypothesis to conclude that since every node in $\left\{\left(\bar{x}_{i}, \bar{x}_{i}^{\prime}\right), x_{i}^{\prime}, d_{i}, y_{i}\right\}$ is clamped while $G_{i}$ is being black pebbled, $\psi\left\lceil\alpha_{i} \cup\left\{\bar{x}_{i}\right\}\right.$ is in QSAT.

The other subcase (ii) is an analogous argument to subcase (i) but for the dual case of a white pebble being discharged from $\bar{x}_{i}^{\prime}$ (rather than it being black pebbled.)

Suppose, on the other hand, that there is a black pebble on $\bar{x}_{i}^{\prime}$ at $t_{11}$. We will now show that only $B_{\alpha_{i}} \cup$ $\left\{x_{i}^{\prime}, d_{i}, y_{i}\right\}$ can be pebbled when we pebble $\bar{x}_{i}^{\prime}$ for the last time before $t_{11}$ at some time $t_{4}$. Suppose for the sake of contradiction that there is a pebble on some other node $z$ at $t_{4}$. Since $y_{i}$ is a predecessor of $\bar{x}_{i}^{\prime}$ and can only be 
pebbled at $t_{2}, y_{i} \in\left[t_{2}, t_{4}-1\right]$. So $d_{i}$ must be empty at $t_{4}-1$ because $\bar{x}_{i}^{\prime}$ has $4 i+2$ predecessors which must be on the graph, along with $z$, at $t_{4}-1$, which fills up the space bound.

In order to pebble $a_{i}$ by $t_{11}$ we must therefore pebble $d_{i}$ at some time between $t_{4}$ and $t_{11}$. Suppose $d_{i}$ is white pebbled. This pebble must be discharged by $t_{11}-1$ because $a_{i}$ has $4 i+1$ predecessors and both $\left(x_{i}^{\prime}, x_{i}\right)$ and $\bar{x}_{i}^{\prime}$ are clamped until $t_{11}$, so $d_{i}$ 's pebble is needed. By frugality there must be a pebble in $H_{i}$ at the time $d_{i}$ is discharged. So at this time there must be pebbles on a node of $H_{i}$, one of $\left(x_{i}, x_{i}^{\prime}\right)$, and $\bar{x}_{i}$ and we must exceed the space bound. Suppose on the other hand that $d_{i}$ is black pebbled between $t_{4}$ and $t_{11}$. This takes $4 i+1$ pebbles and there must be pebbles on $\left(x_{i}, x_{i}^{\prime}\right), \bar{x}_{i}^{\prime}$ and by frugality $z^{\prime}$, where $z^{\prime}$ is between $z$ and $a_{i}$. So we can never pebble $d_{i}$ between $t_{4}$ and $t_{11}$. We therefore know that when $\bar{x}_{i}^{\prime}$ is pebbled for the last time before $t_{11}$, there can be no pebble on $z$.

By the argument which we just finished, any node of $G_{i-1}$ can only be pebbled after $t_{4}$. We now show that $G_{i-1}$ must be simultaneously black pebbled in order to black pebble $a_{i}$.

We know that both $\bar{x}_{i}^{\prime} \in\left[t_{4}, t_{11}\right]$ and $\left(x_{i}^{\prime}, x_{i}\right) \in\left[t_{4}, t_{11}\right]$. Therefore by Lemma 7 , any node in $H_{i}$ can only be pebbled once in $\left[t_{4}, t_{11}\right]$ and must be black. Call the time $h_{i}^{4 i+1}$ is pebbled $t_{10}$, the time $h_{i}^{4 i}$ is pebbled $t_{9}$, the time $h_{i}^{4 i-1}$ is pebbled $t_{8}$, and the time $h_{i}^{1}$ is pebbled $t_{7}$. So $\bar{x}_{i}$ must pebbled at some time $t_{6}$ before $t_{7}$ and $\bar{x}_{i} \in\left[t_{6}, t_{7}-1\right]$. Suppose it is white pebbled. Then it must be discharged before $t_{10}$ because its pebble is needed to pebble $h_{i}^{4 i+1}$. Note that $y_{i}$ must be empty at $t_{7}-1$ since our space bound is reached by $h_{i}^{1}$, s predecessors and the clamping of $\left(x_{i}, x_{i}^{\prime}\right)$ and $\bar{x}_{i}^{\prime}$. So when $\bar{x}_{i}$ is discharged, there can be no pebble on $y_{i}$. Therefore, to discharge $\bar{x}_{i}$, $y_{i}$ must be pebbled again after $t_{7}$ and before $t_{11}$, which is impossible due to its high indegree. Suppose, on the other hand that $\bar{x}_{i}$ is black pebbled at $t_{6}$. This means that $y_{i} \in\left[t_{2}, t_{6}-1\right]$. So there are at least 3 pebbles clamped from $t_{4}$ until $t_{7}-1$. But $d_{i}$ must be pebbled before $t_{7}-1$. So $d_{i}$ must be pebbled before $t_{4}$, at some time $t_{3}$ after $t_{2}$, and $d_{i} \in\left[t_{3}, t_{10}-1\right]$.

Thus $\left\{\left(x_{i}, x_{i}^{\prime}\right), \bar{x}_{i}^{\prime}, d_{i},\left(y_{i}, \bar{x}_{i}\right)\right\} \subseteq\left[t_{4}, t_{7}-1\right]$, so by Lemma 7 any node of $R_{i}$ can only be pebbled black and pebbled once during this interval. Let $t_{5}$ be the time $r_{i}^{1}$ is pebbled. The nodes $G_{i-1} \cup\left\{\left(x_{i}, x_{i}^{\prime}\right), \bar{x}_{i}^{\prime}, d_{i}, y_{i}\right\}$ must all be pebbled at $t_{5}-1$. So $\left\{\left(x_{i}, x_{i}^{\prime}\right), \bar{x}_{i}^{\prime}, d_{i}, y_{i}\right\} \in\left[t_{4}, t_{5}-1\right]$. So $G_{i-1}$ must only be pebbled black and once during this interval, so we can apply the induction hypothesis to conclude that $\psi\left\lceil\alpha_{i} \cup\left\{x_{i}\right\}\right.$ is in QSAT.
Corollary 8: There exists an infinite family of graphs such that any minimal space black-white pebbling of these graphs requires exponential-time, but they can be refuted in linear time with the use of 1 additional pebble.

Proof: Let $\mathcal{G}$ be the DAG corresponding to the formula $\psi=\forall x_{n} \forall x_{n-1} \ldots \forall x_{1}\left(x_{1} \vee \bar{x}_{1} \vee x_{2}\right) \wedge\left(x_{2} \vee \bar{x}_{2} \vee x_{3}\right) \wedge$ $\ldots \wedge\left(x_{n} \vee \bar{x}_{n} \vee x_{1}\right)$. This formula is clearly QSAT, since its $3 \mathrm{CNF}$ part is a tautology. Also, since $\psi$ has $n$ universally quantified variables, by Lemma 5 , the minimal $4 n+3$ pebbling strategy for $\mathcal{G}$ requires time $2^{n}$ to execute. We can pebble $\mathcal{G}$ in linear time using exactly one extra pebble by following the upperbound's strategy except that in each universal widget, we keep a pebble on $x_{i}^{\prime}$ as we pebble $a_{i}$, which then allows us to pebble up the otherside without any repebbling.

\section{Exponential Speedup for Resolution}

In this section we discuss two main results, the PSPACE-completeness of Resolution space, as well as a surprising exponential speedup for Resolution. Due to space limitations, we omit the technical details of the reductions, as well as most of the proofs here but note that they can be found in [8].

Definition 3.1: [1] A configuration $C$ is a set of clauses. If $f$ is a CNF formula, then the sequence of configurations $\pi=C[0], C[1], \ldots, C[k]$ is a RES proof of $C$ from $F$ if $C[0]=\emptyset, C \in \mathcal{C}[k]$, and for each $i<k, C[i+1]$ is obtained from $C[i]$ by one of the following rules: (1) deleting one or more clauses from the current configuration; (2) add the resolvent of two clauses of $C[i]$; (3) download an axiom (clause) of $f$. If $\emptyset \in C[k]$, then $\pi$ is a proof of $f$.

Definition 3.2: [2] The variable space of a proof $\pi$ is the maximum size of any configuration $C$ in $\pi$. The variable space of an unsatisfiable CNF formula $f$ is the minimum space over all proofs of $f$. Unless specified otherwise, space in this paper will refer to variable space. Given a CNF formula $f$ and a number $k$, the Resolution space problem asks whether there is a space $k$ Resolution proof of $f$.

Our first theorem settles the complexity of the Resolution space problem.

Theorem 9: The Resolution space problem is PSPACE-complete. 
Our second theorem, which is quite surprising, shows that allowing (or disallowing) even 3 extra units of storage can have drastic consequences for Resolution-based SAT algorithms.

Theorem 10: There exist CNF formulas such that any minimal space proof of these formulas requires exponential size, but that can be refuted in linear size, with 3 more units of space.

There is a clear connection between the nature of these two results and those of Theorem 1 and Corollary 8. As before, a reduction from the QSAT problem is central to their proofs. The reduction used here is globally quite similar to that of Section 2, but its widgets are very different. Most notably, our construction $\mathcal{G}$ is now a monotone circuit instead of a DAG. But $\mathcal{G}$ still possesses the following two crucial properties.

(1) First, the black-white pebbling number of $\mathcal{G}$ is equal to the black pebbling number of $\mathcal{G}$. That is, our monotone circuit $\mathcal{G}$ has the important property that white pebbles do not help at all, so $\mathcal{G}$ 's optimal pebbling strategy is pure black.

(2) Secondly, if $\psi$ is in QSAT, then any optimal strategy will require exponential time in the number of universal quantifiers. But if we let the strategy use a small constant number of pebbles more, then $\mathcal{G}$ can be pebbled in linear time, whether or not $\psi$ is in QSAT.

Now from $\mathcal{G}$, we define an associated "pebbling formula", $\operatorname{Peb}(G)$, defined below.

Definition 3.3: [2] Let $\mathcal{G}$ be a monotone circuit. $\operatorname{Peb}(\mathcal{G})$ is a set of clauses, with one variable $v_{i}$ for each vertex in $\mathcal{G}$, and containing the following (Horn) clauses: (1) For each source vertex $v$, we have the clause $(v)$; (2) For each AND vertex $v$ with predecessors $u_{1}, \ldots, u_{l}$, we have the clauses $\left(\neg u_{1} \vee \neg u_{2} \vee \ldots \vee u_{l} \vee v\right)$; (3) Finally for each OR vertex $v$ and each predecessor $u$ of $v$, we have the clause $(\neg u \vee v)$. By a Resolution proof of $\operatorname{Peb}(G)$, we mean a Resolution derivation of the unit clause $(s)$ from $\mathrm{Peb}(\mathcal{G})$.

As mentioned earlier, [2] showed that one can extract a black-white pebbling strategy for $\mathcal{G}$ from a Resolution proof of $\operatorname{Peb}(G)$, where the strategy's pebbling number is related to proof's Resolution space. If the converse relationship held, then Theorem $9 \& 10$ would follow easily from Theorem 1 and Corollary 8 . Unfortunately, the converse does not hold and more work is needed. It is not hard to see that from a pure black pebbling strategy for $\mathcal{G}$, we do obtain a corresponding space-preserving Resolution proof of $\mathrm{Peb}(\mathcal{G})$.
Intuitively, since white pebbles do not help to pebble $\mathcal{G}$, by special property (1) of our reduction, the converse relationship should hold for our special graphs, even with the addition of white pebbles.

This is the high-level idea behind the argument. However, significant technical difficulties arise when carrying out the proof. In order to mimic a step of the black pebbling strategy, one of $\mathrm{Peb}(\mathcal{G})$ 's axioms must be downloaded, and this forces the Resolution simulation to use more space than was needed in the pebbling step. When an axiom is downloaded, the Resolution space exceeds the graphs's pebbling number by the axiom's size. The problem with this "slack space" is that some axioms of $\mathrm{Peb}(\mathcal{G})$ are larger than others because their corresponding nodes have higher indegree in $\mathcal{G}$. We therefore exceed the pebbling number by different amounts at different points in the proof. During periods in the proof when only smaller axioms are being downloaded, we are able to use this space difference to deviate from the pure black pebbling strategy. To solve this problem, we further modify $\mathcal{G}$ to obtain $\mathcal{G}$ RES which has even more structure which serves to "fill up" the slack space in $P e b(G$ RES $)$. The simplest way to do this is to allow ourselves the use of OR-nodes, which we use to solve the slack space problem. We also use the OR-nodes to construct efficient quantifier widgets, which reduce the pebbling number of the resulting circuit. Even with this modification, our argument is substantially more complicated than before. As in Section 2, we argue that the only way for the Resolution proof to proceed at each step is to follow the all-black pebbling strategy-however now we need to use a global graph-theoretic argument, whereas before we essentially argued locally.

We show that the QBF formula $\psi$ is in QSAT if and only if $P e b(G \mathrm{RES})$ has a space $6 n+3$ Resolution derivation. Moreover, the above two special properties continue to hold in this context. In particular, our reduction satisfies (2'): If $\psi$ is in QSAT, then any space $6 n+3$ Resolution proof of $\mathrm{Peb}\left(\mathcal{G}_{\mathrm{RES}}\right)$ requires exponential size. However, with $6 n+6$ space, for any $\psi$, the associated formula $\mathrm{Peb}\left(\mathcal{G}_{\mathrm{RES}}\right)$ has a linear-space proof.

Our main results follow from Lemmas $11,12, \& 13$ and Theorem 14. Lemma 11 provides the optimal pebbling strategy for $\mathcal{G}$, while Lemma 12 provides the suboptimal yet linear time strategy. Lemma 13 shows that the there are Resolution proofs whose size and space are proportional to those pebbling strategies.

Lemma 11: If $\psi$ is in QSAT, then the target node $s$ of $\mathcal{G}$ can be pebbled with $3 n+1$ pebbles. 
Lemma 12: There is a black pebbling strategy for $\mathcal{G}$ which pebbles $s$ in time $O(|\mathcal{G}|)$ and uses $3 n+4$ pebbles, regardless of whether $\psi$ is in QSAT.

Lemma 13: The $k$-pebble, $t$-time black pebbling strategies of Lemmas $11 \& 12$ for the target node $s$ of $\mathcal{G}$ imply the existence of space $k+d$ Resolution derivations of $\operatorname{Peb}\left(\mathcal{G}_{\mathrm{RES}}\right)$ which take time polynomial in $t$, where $d$ is the maximum size of any axiom of $P e b\left(G_{\text {RES }}\right)$.

Theorem 14 is the hardest part of the argument. It proves that the Resolution proof obtained from the pebbling strategy from Lemma 11 truly is optimal, and that any space-optimal proof has exponential size.

Theorem 14: Let $\psi$ be a QBF, and let $G_{R E S}$ be the associated monotone circuit. Then if $P e b\left(G_{R E S}\right)$ can be derived using at most $6 n+3$ space, then $\psi$ is in QSAT, and any $6 n+3$ space proof requires $\Omega\left(2^{k}\right)$ steps, where $k$ is the number of universally quantified variables in $\psi$.

We are now able to prove our two main results.

Theorem 9: The Resolution space problem is PSPACEcomplete.

Proof: Every unsatisfiable formula has a space $n$ Resolution proof, and thus there is an NPSPACE algorithm guessing a space $n$ proof. By Savitch's theorem, this implies a PSPACE algorithm. To show PSPACEhardness, from a QBF formula $\psi$, we construct the associated CNF formula Peb(GRES). By Lemmas 11 \& 13 , if $\psi$ is in QSAT, then there is a Resolution derivation of $\operatorname{Peb}\left(\mathcal{G}_{\mathrm{RES}}\right)$ which uses $6 n+3$ space. Conversely, by Theorem 14, if there is a Resolution derivation of $\operatorname{Peb}\left(\mathcal{G}_{\mathrm{RES}}\right)$ using $6 n+3$ space, then $\psi$ is in QSAT.

Theorem 10: There exist CNF formulas which have linear size Resolution proofs that can be verified in space $k+3$, but whose smallest Resolution proofs that can be verified in space $k$ have exponential size.

Proof: Let $\psi=\forall x_{n} \forall x_{n-1} \ldots \forall x_{1} F$ be any totally universally quantified QBF which is in QSAT, and let $\mathcal{G}$ RES be the graph obtained from $\psi$. Since $\psi$ is in QSAT, by Lemmas $11 \& 13$ and Theorem 14, there exist space $6 n+3$ Resolution proofs of $\mathrm{Peb}\left(\mathcal{G}_{\mathrm{RES}}\right)$, and all of them require $\Omega\left(2^{n}\right)$ size. By Lemmas $12 \& 13$, there exists a space $6 n+6$ derivation of $P e b(G$ RES $)$ which only requires $O\left(\left|\operatorname{Peb}\left(\mathcal{G}_{\mathrm{RES}}\right)\right|\right)$ size.

\section{References}

[1] M. Alekhnovich, E. Ben-Sasson, A.A. Razborov, and A. Wigderson. Space Complexity in Propo- sitional Calculus. SIAM Journal of Computing, 31(4):1184 - 1211, 2001.

[2] E. Ben-Sasson. Size Space Tradeoffs For Resolution. STOC, pages 457 - 464, 2002.

[3] S. Cook and R. Sethi. Storage Requirements for Deterministic Polynomial Time Recognizable Languages. JCSS, pages 25 - 37, 1976.

[4] A. Darwiche and D. Allen. Optimal time-space tradeoff in probabilistic inference. In European Workshop on Probabilistic Graphical Models, 2002.

[5] J. Esteban and J. Torán. Space Bounds for Resolution. Information and Computation, 171:84 - 97, 2001.

[6] J. R. Gilbert, T. Lengauer, and R. E. Tarjan. The Pebbling Problem is Complete in Polynomial Space. SIAM Journal of Computing, 9(3):513 524, 1980.

[7] F. Meyer Auf Der Heide. A Comparison of Two Variations of a Pebble Game on Graphs. TCS, 13:315 - 322, 1981 .

[8] P. Hertel and T. Pitassi. An Exponential Time/Space Speedup For Resolution. ECCC TR07-046, 2007.

[9] D. S. Johnson. The NP-Completeness Column: An Ongoing Guide. J. Algorithms, 4(4):397-411, 1983.

[10] B. Kalyanasundaram and G. Schnitger. On the power of white pebbles. In STOC, pages 258-266, 1988.

[11] A. Lingas. A PSPACE-Complete Problem Related to a Pebble Game. In ICALP, pages $300-321$, 1978.

[12] J. Nordström. Narrow Proofs May Be Spacious: Separating Space and Width in Resolution. STOC, 2006.

[13] N. Pippenger. A Time-Space Trade-Off. JACM, pages 509-515, 1978.

[14] N. Pippenger. Pebbling. TR RC8528, IBM Watson Research Center, 1980.

[15] R Wilber. White pebbles help. In STOC, pages 103-112, 1985. 